\title{
Ovarian Mucinous Cystadenoma with Anaplastic Carcinoma Arising in The Mural Nodule:A Case Report
}

\author{
Bodepudi Srija*, Susruthan M. and Barathi G. \\ Department of Pathology, Sri Ramachandra Medical College and Research Institute, Porur, Chennai-, India
}

\section{ABSTRACT}

Mural nodules in cystic ovarian neoplasms are very rare. Mural nodules are localized thickened nodular areas occurring in the wall of cystic mucinous or serous tumors of the ovary. Histologically they may be benign reactive sarcoma like lesions or malignant lesions like sarcomas and anaplastic carcinomas. We report a case of mural nodule of anaplastic carcinoma in a mucinous cystadenoma of ovary.

Keywords: MucinousCystadenoma, Mural Nodule, Anaplastic Carcinoma.

\section{Introduction}

A mural nodule in a cystic ovarian neoplasm is an extremely rare event with an incidence between 2 to 5 per million. The mural nodules are classified histologically as benign and malignant lesions. A wide variety of mural nodules, such as anaplastic carcinoma, sarcoma, sarcoma-like mural nodule, and other benign neoplasms like leiomyoma and rhabdomyoma have been reported. The background ovarian neoplasm is a cystic tumor, either a mucinous cystic tumor or a serous cystic tumor with the former accounting for the majority of the cases. The subject of this report was a mural nodule with an anaplastic carcinoma in a mucinous cystadenoma.

\section{Case Report}

A 43 year old woman was admitted in the department of Obstetrics and Gynecology, Sri Ramachandra Medical College with a complaint of lower abdominal pain and abdominal distension. Examination showed a palpable, non-tender suprapubic mass which was firm in consistency. MRI abdomen revealed an abdominopelvic cystic lesion probably arising from the left ovary measuring $19 \times 15 \times 9.9 \mathrm{~cm}$ with a solid component. Uterus was unremarkable. The patient was planned for staging exploratory laparotomy. Intra operatively a left ovarian mass was noted adherent to the abdominal wall and bowel. A malignant tumor of ovary with possibility of germ cell tumor/ high grade surface epithelial tumor associated with mucinous cystadenoma was diagnosed during the operation by frozen section. Patient underwent total hysterectomy with bilateral salpingo oophorectomy. Bilateral pelvic and para aortic lymph node dissection was performed. Peritoneal wash fluid was obtained. Post-operative period was uneventful.
Gross examination of the ovarian mass showed a cystic grey white mass with intact capsule, measuring 19x14x10cm. External surface was nodular, grey white in color. Cut section exuded colorless mucoid material. Tumor showed predominantly cystic component $(80 \%)$ with focal solid area $(20 \%)$. Cystic component was multilocular and all the locules were grey white with a smooth lining. The solid areas in the wall showed multiple firm, grey white nodules with focal hemorrhages and largest nodule measured $5 \times 5 \times 3 \mathrm{~cm}$ (Fig.1). Attached fallopian tube was $5 \mathrm{~cm}$ in length with a small paratubal cyst. Right adnexa and uterus were unremarkable.

Microscopic examination of the cystic areas showed ovarian tissue lined by a single layer of tall columnar mucin rich epithelium. The solid mural nodule showed diffuse sheets of atypical cells with rhabdoid differentiation. Individual tumor cells were polygonal with abundant amount of eosinophilic cytoplasm. The nucleus was eccentrically placed with irregular margins, vesicular chromatin and prominent nucleoli. Few of the atypical tumor cells showed multinucleation. Plenty of atypical mitosis were seen. Areas of hemorrhage and necrosis were noted (Fig.2). 22 lymph nodes were submitted, all free of tumor. Peritoneal wash fluid was positive for malignancy. Pathological staging was pT1c3 pN0 cM0.

Immunohistochemistry for Vimentin, Cytokeratin and CD30 was done to confirm anaplastic carcinoma and to exclude embryonal carcinoma. The atypical pleomorphic cells in the solid areas showed diffuse cytoplasmic positivity for vimentin and cytokeratin (Fig.3). CD30 was negative.

A diagnosis of mucinous cystadenoma with anaplastic carcinomatous mural nodule was made. 


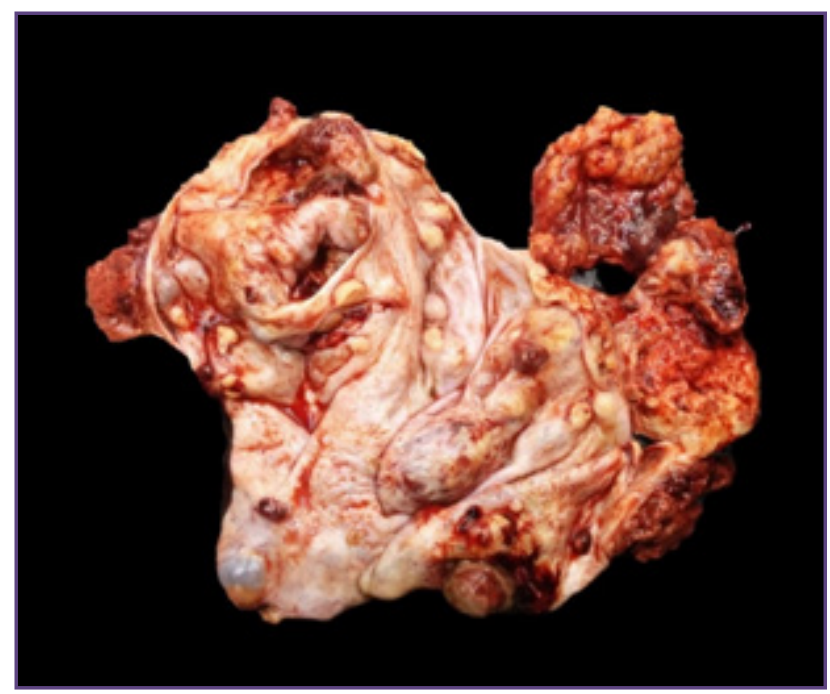

Fig. 1: Cut section of ovarian cyst with multiple mural nodules (20X) H \& E.

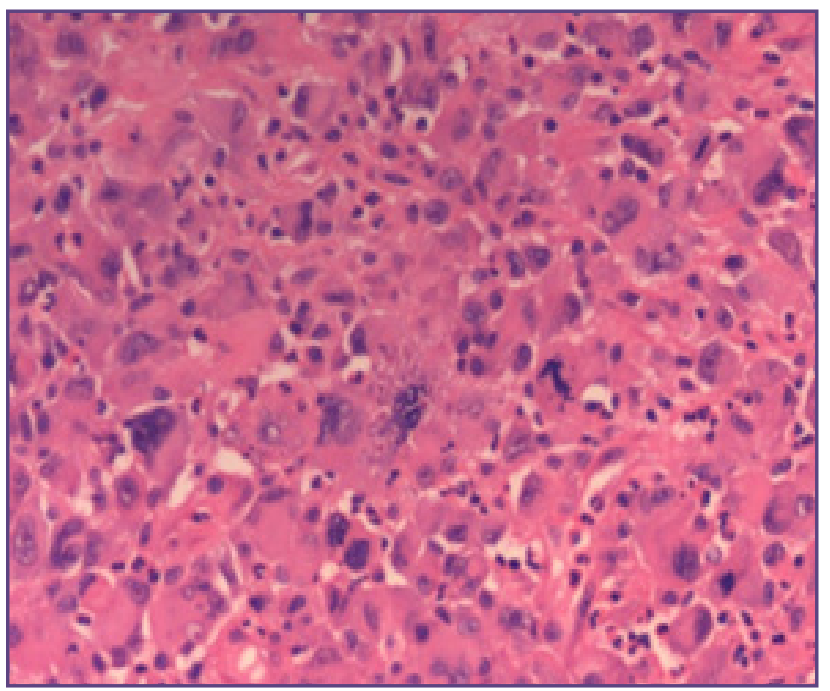

Fig. 2: Pleomorphic cells with abundant eosinophilic cytoplasm, eccentric nucleus, vesicular chromatin and prominent nucleoli. (400X) H\&E

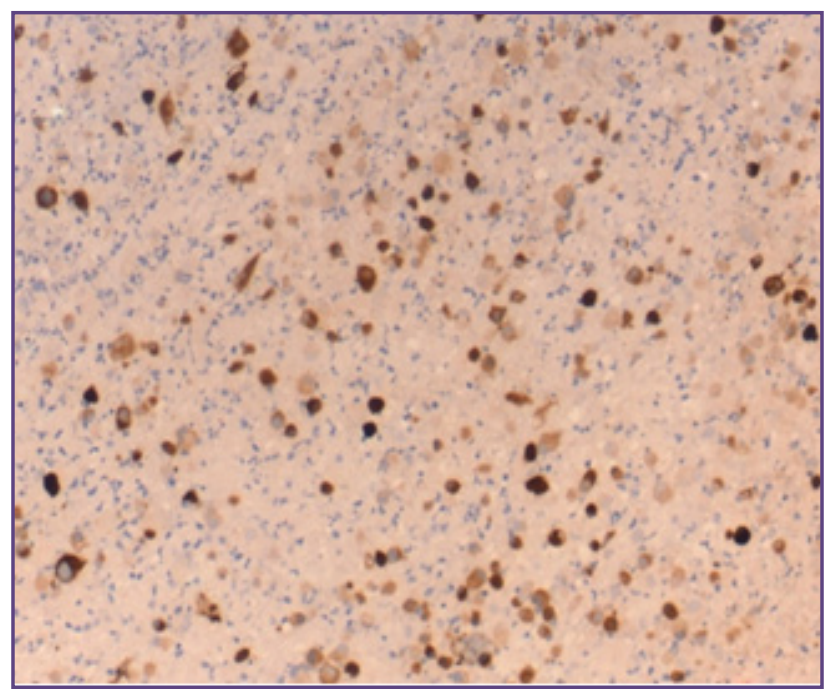

Fig. 3: Cytokeratin stain showing positive staining of pleomorphic Cells (100X) IHC.

\section{Discussion}

Mural nodules are localized single or multiple nodular areas in the cyst wall of cystic tumors of the ovary. Majority of the nodules are usually seen in the mucinous tumors with a few occurring in the serous counterparts. The mural nodules may be benign or malignant. The benign variant of mural nodule is termed as reactive sarcoma like lesion. These nodules present as well circumscribed small lesions with sheets of spindle shaped cells along with plenty of multinucleate giant cells and inflammatory cells. Occasional mitotic figures may be seen. Vascular and stromal infiltration is not present. ${ }^{[1]}$ The cause of these lesions has been attributed to reactive change secondary to hemorrhage into the cyst wall by Prat and Scully, who described this lesion for the first time in 1979). ${ }^{[2]}$ Rarely true benign tumors like a Leiomyoma ${ }^{[3]}$ or Rhabdomyoma ${ }^{[4]}$ may present as a mural nodule.

The malignant counterpart of the mural nodules may present as a sarcoma or as an undifferentiated anaplastic carcinoma or a combination of both. Majority of the reported malignant lesions are sarcomas with fibrosarcoma, rhabdomyosarcoma, carcinosarcoma and undifferentiated sarcoma being reported. Anaplastic carcinomatous mural nodules are rare with only few reported in the literature. Most of these lesions occur in the background of either borderline 
mucinous tumor or mucinous cystadenocarcinoma with only very few occurring in benign mucinous cystadenoma. Histologically anaplastic carcinomatous nodules present as a rhabdoid or spindle cell pattern. The rhabdoid form presents as diffuse sheets of cells with abundant eosinophilic cytoplasm and eccentric oval nucleus whereas the spindle cell pattern presents as sheets of spindle cells with scanty cytoplasm and vesicular nuclei. A combination of both the patterns may exist which is termed as pleomorphic pattern. ${ }^{[5,6,7,8]}$ The malignant tumors have ill-defined borders and invade vascular structures and capsule unlike the benign counterparts. Rarely the nodule may also present as a clear cell carcinoma. ${ }^{[9]}$

The pathogenesis of the nodule in mucinous tumors remains unclear, but several hypotheses exist. Desouki ${ }^{[10]}$ proposed that the concept of a collision tumor, which is the result of a collision between two neoplasms that have arisen in adjacent areas, might be the best explanation for the pathogenesis. Another hypothesis proposed the nodule is formed as a result of progressive dedifferentiation of mucinous cells, with a concomitant loss of the ability to produce mucin. The possible presence of totipotent stem cells in the stroma of mucinous tumors and their malignant transformation has also been proposed as another pathogenetic mechanism for tumor genesis. Further studies are necessary before the pathogenesis of this lesion can be validated.

Immunohistochemically the anaplastic carcinomas show strong cytoplasmic positive expression of Pancytokeratin, AE1/AE3 and EMA in contrast to focal and weak expression in reactive sarcoma like nodules.

Positive vimentin expression is attributed to dedifferentiation of the tumor. Even the spindle cell variant of anaplastic carcinoma express strong cytoplasmic expression of cytokeratin which is helpful in differentiating these tumors from the sarcomas and the benign reactive sarcoma like mural nodule.

The malignant mural nodules are aggressive tumors with a bad prognosis and a survival rate of $50 \%$. However when diagnosed in the early stage (Stage I) these tumors have a reasonably good prognosis. Further when these malignant mural nodules arise in a mucinous cystadenoma they have a better outcome than when they arise in a mucinous cystadenocarcinoma

\section{Conclusion}

In conclusion, anaplastic carcinoma mural nodules in a benign mucinous epithelial tumor are very rare. Careful gross examination and extensive sampling is very important to identify these malignant lesions before giving a diagnosis of benign cystic epithelial tumor, as a diagnosis of coexistent anaplastic carcinoma mural nodules have a bad prognosis and need aggressive therapy and close follow up.

\section{References}

1. Bague S, Rodriguez IM, Prat J. Sarcoma-Like Mural Nodules in Mucinous Cystic tumors of ovary revisited: a clinicopathologic analysis of 10 additional cases. Am J Sur Pathol. 2002;26:1467-76.

2. Prat J, Scully RE. Ovarian mucinous tumours with sarcomalike mural nodules: a report of seven cases. Cancer. 1979;44:1332-44.

3. Nazmoon L, Sureshni I.J. Spectrum of mural nodules in mucinous cystic tumours of ovary (MCTO): A rare case of an ovarian mucinous cystadenoma with a mural leiomyoma. Pathology. 2014;46:S70.

4. Huang TY, Chen JT, Ho WL. Ovarian serous cystadenoma with mural nodules of genital rhabdomyoma. Hum Pathol. 2005;36:433-35.

5. Provenza C, Young RH, Prat J. Anaplastic carcinoma in mucinous ovarian tumors: a clinicopathologic study of 34 cases emphasizing the crucial impact of stage on prognosis, their histologic spectrum, and overlap with sarcomalike mural nodules. Am J Surg Pathol. 2008;32:383-9.

6. Prat J, Young RH, Scully RE. Ovarian mucinous tumours with foci of anaplastic carcinoma. Cancer. 1982;50:300-4.

7. Chan Y F, Ho HC, Yau SM, Ma L. Ovarian mucinous tumor with mural nodules of anaplastic carcinoma. Gynecol Oncol. 1989;35:112-9.

8. Yamazaki H, Matsuzawa A, Shoda T, Iguchi H, Kyushima N. Ovarian mucinous cystic tumor of borderline malignancy with a mural nodule of anaplastic spindle cell carcinoma: a case report. J Ovarian Res. 2013;6:86.

9. Allende DS, Drake RD, Chen L. Mural Nodules of Clear Cell Carcinoma in a Mucinous Borderline Tumor of the Ovary: A Case Report. Patholog Res Int. 2010;2010:438534.

10. Desouki MM, Khabele D, Crispens MA, Fadare O. Ovarian mucinous tumor with malignant mural nodules: dedifferentiation or collision tumors. Int J Gynecol Pathol 2015;34:19-24.

*Corresponding author:

Dr Bodepudi Srija., Post Graduate, Department of Pathology, Sri Ramachandra Medical College, Porur, Chennai, India600116

Phone: +91 7702020527

Email: bodepudisrija@gmail.com

Financial or other Competing Interests: None. 